\title{
A novel cutting tool design to avoid surface damage in bone machining
}

\begin{abstract}
With its anisotropic structure, bone machining occurs as shear/serrated cutting mechanisms at low values of uncut chip thickness while at high values it results in fracture cutting mechanisms which lead to significant tissues damages; hence, utilising conventional tools at high material removal rates comes with drawback on surface damages, situation that needs to be avoided. This paper reports on a novel design of a milling cutter which includes on the back of main cutting edge a succession of micro-cutting edges arranged on an Archimedes spiral that allows the limitation of surface damage. That is, by adjusting the feed rate, this tool design allows the change of the cutting mechanism as follows: (i) "shear/serrated" cutting mode: when the feed rate is smaller than a pre-established threshold, only the main cutting edges work which yields a shear/serrated cutting mechanism; (ii) combined "fracture \& shear" cutting mode occurring at high feed rate caused by: the main cutting edges working in fracture cutting mechanism while the subsequent micro-cutting edges work under shear cutting mechanism, combination which leads to significant reduction of bone surface damages. This new tool concept was materialised on a solid diamond composite, characterised by excellent heat conduction and low wear rates. Cutting experiments with various values of feed rates showed that the proposed tool designed concept significantly reduced the fracture damage of bone cut surface as well as cutting temperature compared with the dimensionally equivalently conventional tool.
\end{abstract}

Keywords: Bone cutting; novel tool design; surface integrity. 


\section{Introduction}

The main constituent to be cut when performing bone surgery, cortical bone, is an anisotropic tissue similar to fibre reinforced composite which mainly consists of osteons (fibres) and interstitial lamellae (matrix), while resulting in high structural density. Thermal necrosis [1], tool wear/breakage [2], loss of cutting precision [3] and structural/mechanical damage on the living "workpiece" could occur in bone cutting when performing surgical interventions. It has been reported that for implant surgical procedures such as arthroplasty, in which the bone needs to be cut to fit the shape of an implant, cutting at high accuracy and at low temperatures is needed to ensure the success of operation and faster patient recovery [4]. Nevertheless, some challenges exist in bone cutting. As living material, the bone cells begin to die when the temperature rises above $55^{\circ} \mathrm{C}$ for a period longer than $30 \mathrm{~s}$ [5], that can occur during bone surgery. On the other hand, while showing a semi-brittle behaviour with anisotropic structure, fracture damage can easily occur in bone cutting causing damages of the surrounding tissues while leading to a poor surface quality which can slow patient's post-operation recovery [6,7]; the morphologies and formation mechanisms of the chip have been found to vary when changing the uncut chip thickness (UCT) in bone

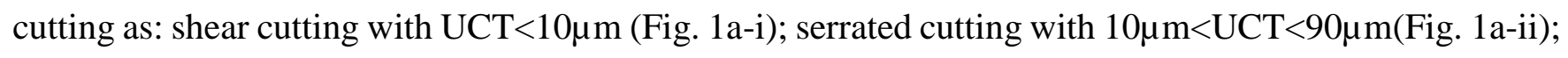
fracture cutting with $90 \mu \mathrm{m}<\mathrm{UCT}$ (Fig. 1a-iii) [6]. As fracture has the tendency to propagate inwards from the cut surface, and it is difficult to control its initiation, the cutting quality is mainly dependent on the surgeon's technique.

On the other hand, the cutting tools currently used in surgical interventions are similar in concept and geometries to those developed for metal cutting. Thus, as in metal cutting, machining complex features in bone while achieving simultaneously various process objectives (e.g. high cutting efficiency/precision, workpiece surface quality, and low levels of cutting force, temperatures, and tool wear rates) is always difficult [8-11]. For example, in bone milling, to generate a smooth surface the feed rate needs to be small enough to make the chip formation under the shear/serrated cutting mechanism, but this also leads to low material removal rate lengthening the intervention time and increasing the risk of necrosis [12]. Moreover, it is difficult for the surgeon to control manually the feed rate at low values and it often occurs that the cutting could be performed in fracture mode (due to accidental increase of feed rate) situation that leads to surface damage. Hence, specialist cutting tool that could ensure the avoidance of workpiece surface damage even at high feed rates while shortening the intervention time is of high importance.

The commonly used cutting tools in bone surgery are generally manufactured from stainless steel characterised by low wear resistance and thermal conductivity. The low thermal conductivity leads to 
temperature increase at the tool-workpiece interface with inherent possibility of damaging the surrounding tissues; additionally, tool wear is also reported to increase the cutting forces when bone machining which could lead to severe surface damage and high cutting temperature [10]. To avoid this, the cutting tools could be manufactured of solid diamond that offers significant wear resistance [13] while providing rapid heat transfer from the cutting zone due to its high thermal conductivity [14]. Furthermore, since the cutting tools need to be repeat sterilised and work under flood saline irrigation, the solid diamond, with its high corrosion resistance, notable mechanical integrity and evident biocompatibility, is foreseen to be an advantage as a tool material $[15,16]$. Thus, it could be considered that using diamond as tool material in bone cutting would bring an improved performance in surgery.

In this context, to improve the surface quality while reducing cutting time and temperature in bone cutting, this paper reports on a novel tool design which can be utilised in a dual mode depending on the feed rate. That is, the proposed milling tool could work: (i) "shear/serrated" cutting mode when the feed rate is smaller than a pre-established threshold, where only the main cutting edges work and yielding a shear/serrated cutting mechanism; (ii) combined "fracture \& shear" cutting mode at high feed rate where the main cutting edges work in fracture cutting mechanism while the subsequent micro-cutting edges work under shear cutting mechanism. This design allows the bone surgery works out a smooth cut surface under both low and high feed rates. The tool geometry is designed by including on the back of a main cutting edge an array of micro-cutting edges, arranged on an Archimedes spiral, to perform a succession microcuts. An analytical model of uncut chip thickness is proposed to assist the design of the cutting teeth while a prototype was manufactured from a solid diamond structure with its excellent heat dissipation and low wear rate properties. To demonstrate the performance of the novel tool design, bone milling tests have been performed while the morphology/integrity of the cut surface and cutting temperature were evaluated and compared with the results obtained from conventional milling cutters, thus proving the efficiency of the proposed tool.

\section{Tool design for controlled bone cutting mechanism}

\subsection{Tool design concept with controlled bone cutting mechanism}

The design novelty of the proposed bone cutting tool is to switch between shear/serrated (main cutting edge working only) and combined fracture $\&$ shear cutting (main and micro-cutting edges working successively) modes depending on the value of the feed rate, as shown in Fig. 1(a) and (b). This design consists of a special curve on the back on the main cutting edge on which an array of micro-cutting edges 
are included for performing additional finishing function thus, allowing the tool to operate in two different working modes.

For this purpose, an Archimedes spiral (Fig. 1c) is employed to ensure equal radial pitch (difference between start and end of the spiral $-P$ ) for the backs of the main cutting edges where the micro-cutting abrasives are uniformly distributed, as shown in Fig. 1(d). With this concept, a uniform spatial distribution of micro-cutting edges is obtained, with the radius of flank face, belonging to the Archimedes spiral, of each micro-edge (Fig. 1d) being expressed as in Eq. 1.

$$
R_{\vartheta}=R_{m}-\frac{P \times \vartheta}{2 \pi}
$$

where $R_{\vartheta}$ is the tool radius of a micro-cutting edge at position of angle $\vartheta, P$ is the spiral pitch and $R_{m}$ is the tool radius of main cutting edge.

With this geometry, the proposed tool can work under two different modes:

(i) Shear/serrated cutting mode - Since the radius of micro-cutting edges $\left(R_{\vartheta}\right)$ decreases following an Archimedes spiral, when the feed rate is under critical value (yielding a feed length shorter than the radius reduction of the spiral), these micro-cutting edges located on the flank surface would not cut the workpiece and therefore, only main cutting edge is in cutting condition (Fig. 1b-i). This critical value of the feed rate, which is related to the spiral pitch and will be estimated in paragraph 6 , would be set to match with the threshold of transition between serrated cutting and fracture cutting mechanisms of the bone [6]; thus, when the feed rate is under the critical value shear/serrated cutting mode (Fig. 1a-i and ii) could be achieved by main cutting edge while the micro-cutting edge not working.

(ii) Combined fracture \& shear cutting mode - when the value of feed rate is higher than the critical value, the main cutting edge works under fracture cutting mechanism (Fig. 1a-iii) which can damage the surface quality but the subsequent micro-cutting edges will work under shear cutting mechanism (Fig. 1a-i) which providing a finishing action thus, allowing the "recovery" of workpiece quality (Fig. 1b-ii).
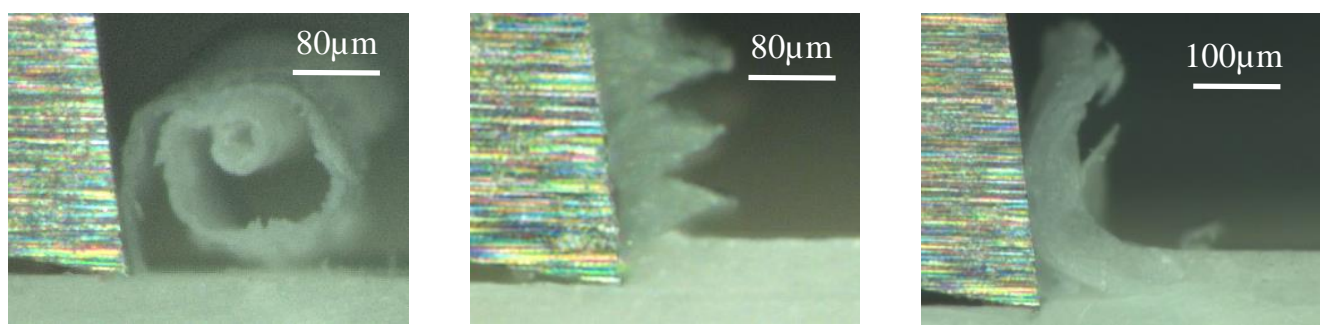
(a-i)



$(b-i)$



(c) (a-ii)

(a-iii)

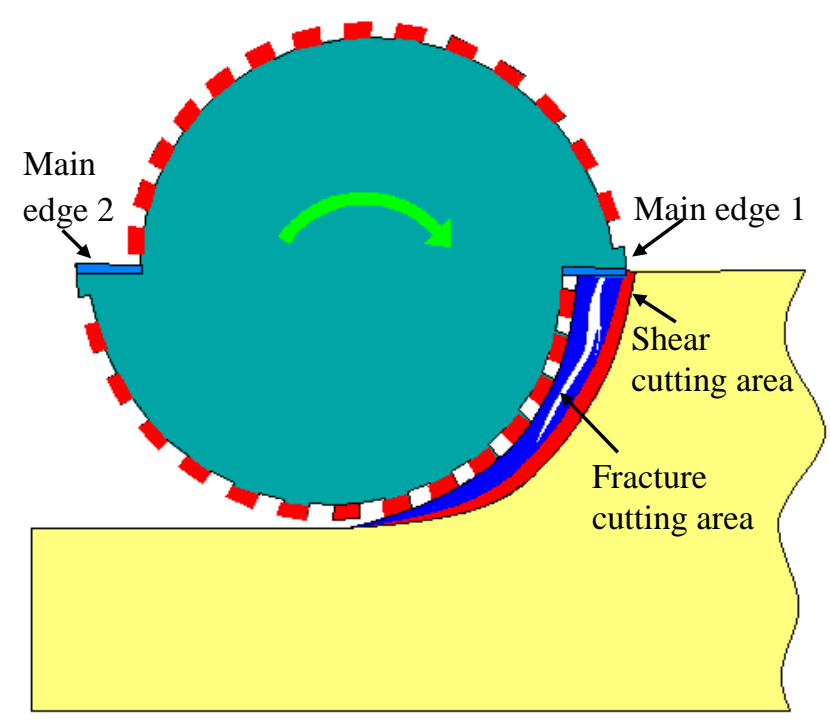

(b-ii)

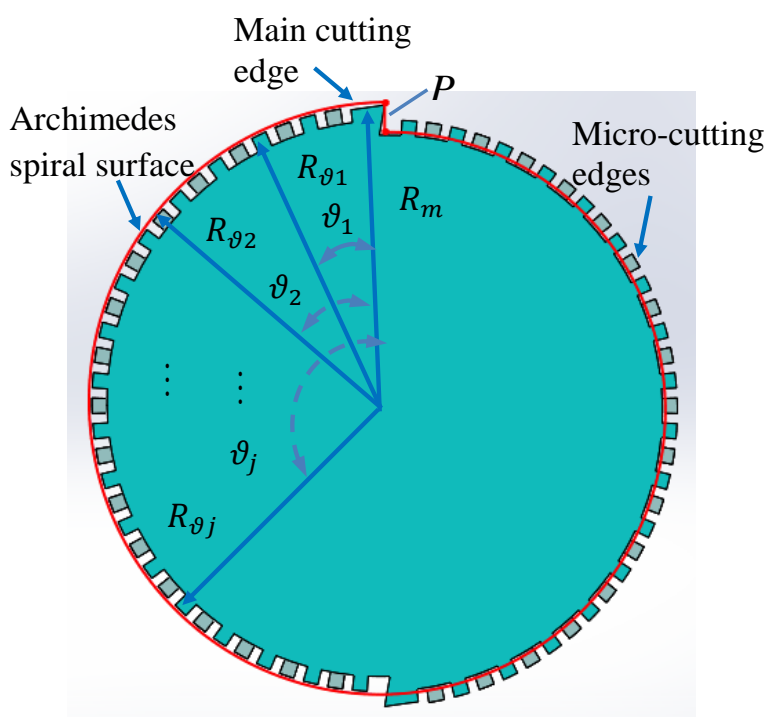

(d)

Fig. 1. Proposed tool design concept: bone cutting mechanism [6] under shear (a-i) serrated (a-ii) and fracture cutting (a-iii); proposed tool working modes within shear/serrated cutting mode ( $b-i)$ and combined fracture \& shear cutting mode(b-ii); employed Archimedes spiral for tool design (c) and its materialisation on proposed novel tool (d).

Thus, regardless of the variation of cutting condition imposed by manual operation of the surgeon, the novel tool design ensures always a reduced damage of bone surface because: (i) at low feed rate yields "shear/serrated" cutting mode resulting in good surface quality; (ii) at high feed rate yields combined "fracture \& shear" cutting mode which also ensures good surface quality. 


\subsection{Geometrical characteristics of the proposed tool}

To evaluate the threshold of feed rate for the transition between main edge only and the main \& micro-cutting edges combined working modes of proposed tool, the uncut chip thicknesses of both main and micro cutting edges need to be calculated. However, the traditional circular trajectory assumption based uncut chip thickness approximation model $\left(h=f_{t} \sin \theta\right)$ cannot precisely describe the actual chip formation as the trajectory of milling cutting edge is a trochoid that results from the translation and rotation of the tool $[17,18]$. To get the accurate and uniform value of the uncut chip thickness for each cutting edge in the design of the proposed tool geometry, Martellotti's estimation approach [19] could be applied and the uncut chip thickness of the micro-cutting edge $i$ at rotation angle $\theta$ can be calculated as:

$$
h_{m i c}^{i}(\theta)=R_{i}-\sqrt{R_{i-1}^{2}-\left(\frac{V_{f} \delta \cos \theta}{2 \pi n}\right)^{2}}+\frac{V_{f} \delta \sin \theta}{2 \pi n}
$$

where $n$ is the tool rotational speed, $V_{f}$ is the feed speed, $\delta$ is the interval angle between two micro-cutting edges, $0<i \leq N, N$ is the number of whole micro-cutting edges on the back of one main cutting edge.

From Eq. (1), the radius of the micro-cutting edge $i$ can be expressed as

$$
R_{i}=R_{m}-\frac{i P \delta}{2 \pi}
$$

Then the maximum uncut chip thickness (MUCT) of a micro-cutting edge $i$ can be expressed as:

$$
h_{\text {micmax }}^{i}=h_{\text {mic }}^{i}\left(\theta_{d}\right)=R_{m}-\frac{i P \delta}{2 \pi}-\sqrt{\left(R_{m}-\frac{(i-1) P \delta}{2 \pi}\right)^{2}-\left(\frac{V_{f} \delta \cos \theta_{d}}{2 \pi n}\right)^{2}}+\frac{V_{f} \delta \sin \theta_{d}}{2 \pi n}
$$

where $\theta_{d}$ is the equivalent angle of depth of cut $(d)$ which can be calculated as

$$
\theta_{d}=\arccos \left(\frac{R_{m}-d}{R_{m}}\right)
$$

The micro-cutting edges (situated on the Archimedes spiral) start to work only when the MUCT $h_{\text {micmax }}^{i}>0$. Since the difference of uncut chip thicknesses for each micro-cutting edge is very small and their values can be treated as uniform, the transition from main edge only cutting to combined cutting modes can be estimated from the limit condition of $h_{m i c}^{1}\left(\theta_{d}\right)=0$. Thus, the critical value of feed rate can be estimated as:

$$
f_{z T}=\frac{1}{z}\left(P-\frac{2 \pi R_{m}}{\delta}\right) \sin \theta_{d}+\frac{1}{z} \sqrt{\left(\frac{2 \pi R_{m}}{\delta}-P\right)^{2} \sin ^{2} \theta_{d}-P^{2}+\frac{4 \pi R_{m} P}{\delta}}
$$


where $z$ is the number of main cutting teeth.

Thus, the main \& micro-cutting edges combined working mode can be estimated to be active when the combined feed per tooth, $f_{z c}$ (including one main cutting edge and its corresponding micro-cutting edges arrayed on its back), is larger than the threshold $\left(f_{z c}>f_{z T}\right)$. For instance, under the radial depth of cut equal to tool radius condition $\left(\theta_{d}=90^{\circ}\right)$ the combined feed per tooth should meet the condition of $f_{z c}>$ $P / z$ to make the tool work under main $\&$ micro edges combined cutting mode.

This can be also explained as in Fig. 2(a): the main cutting edge enters the workpiece at time $t_{1}$ and rotates to the position in angle $\delta$ at time $t_{2}$ where the entrance cutting edge switch to the first micro-cutting edge with the radius reduction of $d_{2}=R_{\mathrm{m}}-R_{1}=P \delta / 2 \pi$, while at this time period the tool feeds forward with the distance of $d_{1}=V_{f} \delta / 2 \pi n$. In this condition, to make the first micro-cutting edge cut into the workpiece, the feed length $\left(d_{1}\right)$ for one edge rotation needs to be larger than the spiral radius decrease of the tool $\left(d_{2}\right)$ as

$$
d_{1}>d_{2} \Rightarrow \frac{V_{f} \delta}{2 \pi n}>\frac{P \delta}{2 \pi} \Rightarrow f_{z c}>\frac{P}{Z}
$$



(a) 


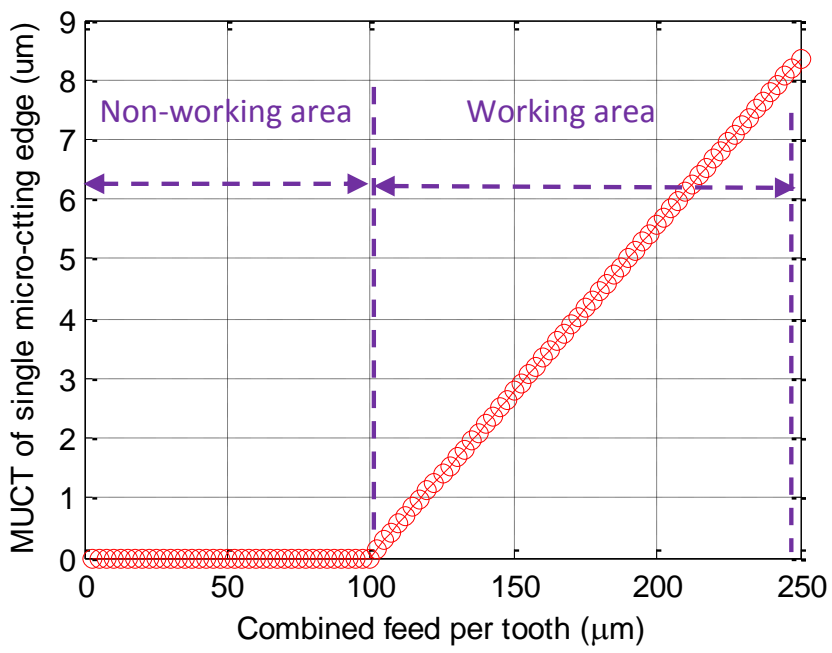

(b)

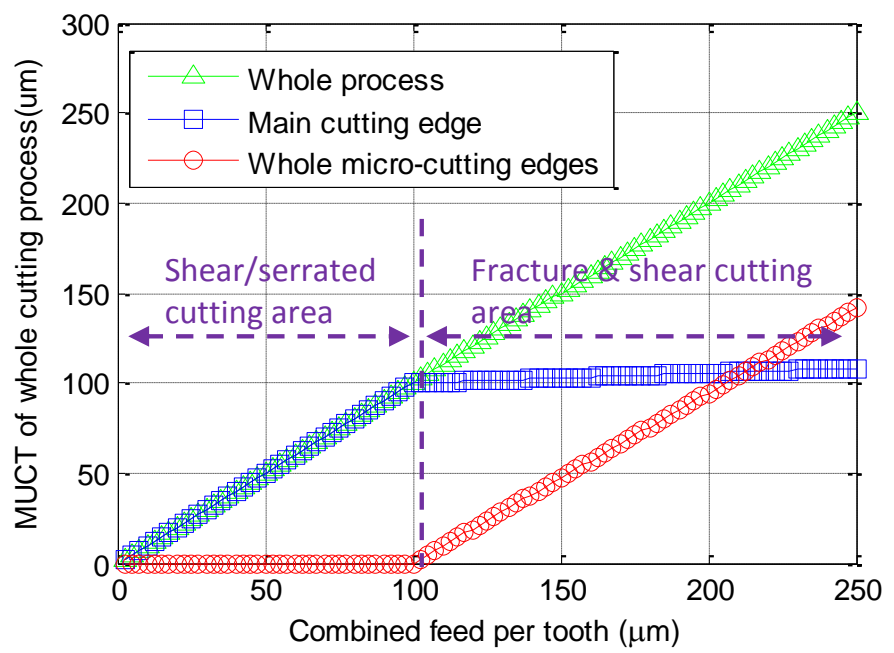

(c)

Fig. 2. Tool working mechanism and relationship between MUCT and combined feed per tooth: (a) working mechanism of proposed tool, (b) MUCT of single micro-cutting edge and (c) MUCT of whole cutting process.

To make sure the transition between main edge only and main \& micro edges combined cutting modes of proposed tool synchronises with the transition of bone cutting mechanism from serrated cutting to fracture cutting, the MUCTs of both micro-cutting edges $\left(h_{\text {micmax }}^{i}\right)$ and main cutting edge $\left(h_{\text {macmax }}\right)$ need to be calculated for assisting the selection of the parameters of tool geometry. According to former analysis, under main edge only cutting mode, the MUCTs can be expressed as:



And under main \& micro edges combined cutting mode the MUCTs can be expressed as:

$$
\left\{\begin{array}{l}
h_{\text {micmax }}^{i}=R_{m}-\frac{i P \delta}{2 \pi}-\sqrt{\left(R_{m}-\frac{(i-1) P \delta}{2 \pi}\right)^{2}-\left(\frac{z f_{z c} \delta \cos \theta_{d}}{2 \pi}\right)^{2}}+\frac{z f_{z c} \delta \sin \theta_{d}}{2 \pi} \\
h_{\text {macmax }}=R_{m}-\sqrt{\left(R_{m}\right)^{2}-\left(f_{z c} \cos \theta_{d}\right)^{2}}+f_{z c} \sin \theta_{d}-\sum_{i=1}^{N} h_{\text {micmax }}^{i}
\end{array} f_{z c}>f_{z T}\right.
$$

According to previous research in bone orthogonal cutting [6], the critical UCT value of the transition from shear cutting to serrated cutting $\left(h_{s c}\right)$ is about $10 \mu \mathrm{m}$ and from serrated cutting to fractured cutting $\left(h_{f c}\right)$ is about $90 \sim 100 \mu \mathrm{m}$. Based on this, when selecting the threshold $\left(f_{z T}\right)$ and the interval angle between two micro-cutting edges $(\delta)$, the MUCTs of micro-cutting edges $\left(h_{\text {micmax }}^{i}\right)$ and main cutting 
edge ( $\left.h_{\text {macmax }}\right)$ need to meet the condition of Eq. (10): Eq. (10-1) refers to the condition of main cutting edge to work only in shear/serrated cutting mode while the micro-edges do not contribute to cutting; Eq. (10-2) refers to the conditions that the main cutting edge works in fracture mode while the micro-edges work in share mode; this leads to the combined "facture \& share" working mode of the cutter.

$$
\left\{\begin{array}{lrr}
h_{\text {macmax }}\left(f_{z T}\right)<h_{f c} & \text { (under shear/serrated cutting mode) } & (10-1) \\
h_{\text {micmax }}^{i}\left(f_{z c}, \delta\right)<h_{s c} & \text { (under combined fracture \& cutting mode) } & (10-2)
\end{array}\right.
$$

Thus, for a prototype tool of $\phi 4 \mathrm{~mm}$ with two main cutting edges and working under $\theta_{d}=90^{\circ}$, according to Eq. (6) and (10), the spiral pitch $P$ then, can be selected as $200 \mu \mathrm{m}$ and the interval angle between two micro-cutting edges, $\delta$, as $10^{\circ}$ with the result of $f_{z T}=100 \mu \mathrm{m}$. With this geometry, the relationship between MUCTs and the feed rate can be predicted with an example given in Fig.2 (b) and (c), where the tool working mode is: (i) if $f_{z C}<f_{z T}$ the tool works on shear/serrated cutting (main edge only cutting) mode; (ii) if $f_{z C}>f_{z T}$ it would work with combined fracture \& shear cutting (main \& micro edges combined cutting) mode. Thus, when works at high feed rates, the proposed tool, on one hand, could reduce risk of the fracture damage generation due to the decrease of main cutting edge MUCT (Fig. 2c) while, one the other hand, could stop the possible fracture from propagating inwards the workpiece and "recover" the surface due to the shear cutting mechanism of micro-cutting edge (Fig. 2b). This would not only allow the tool working in high feed rate which yield high cutting efficiency and low temperature but also reduce the risk of surface damage when a surgeon increases the feed rate by mistake.

\subsection{Tool fabrication}

Fig. 3(a) presents the prototype design of this cutting tool concept with the following main parameters: diameter $4 \mathrm{~mm}$; spiral pitch for micro-cutting edges $0.2 \mathrm{~mm}$; two main cutting edges with rake angle $\gamma_{\text {main }}=10^{\circ}$, clearance angle $\alpha_{\text {main }}=5^{\circ}$, helix angle $\lambda_{\text {main }}=0^{\circ}$, width $3.2 \mathrm{~mm}$; and micro-cutting edges with length $100 \mu \mathrm{m}$, depth $100 \mu \mathrm{m}$, width $0.8 \mathrm{~mm}$, number $34 \times 4$, interval angle $\delta=10^{\circ}$, rake angle $\gamma_{\text {micro }}=5^{\circ}$, clearance angle $\alpha_{\text {micro }}=5^{\circ}$, helix angle $\lambda_{\text {micro }}=0^{\circ}$.

The proposed tool was fabricated of a solid polycrystalline diamond (PCD) considering the advantages offered by excellent thermal conductivity and high hardness. To generate these main and micro-cutting edges, four slices of PCD with the thickness of $0.8 \mathrm{~mm}$ were fabricated by EDM (Sodick AP200L; wire diameter $=0.1 \mathrm{~mm}$ ) and mounted to a stainless steel shaft, as shown in Fig. 3(b) and (c). These main and micro-cutting elements were generated individually with staggered array around the 
periphery of the diamond structure following the Archimedes spiral, where the profile of each cutting edge was well defined.

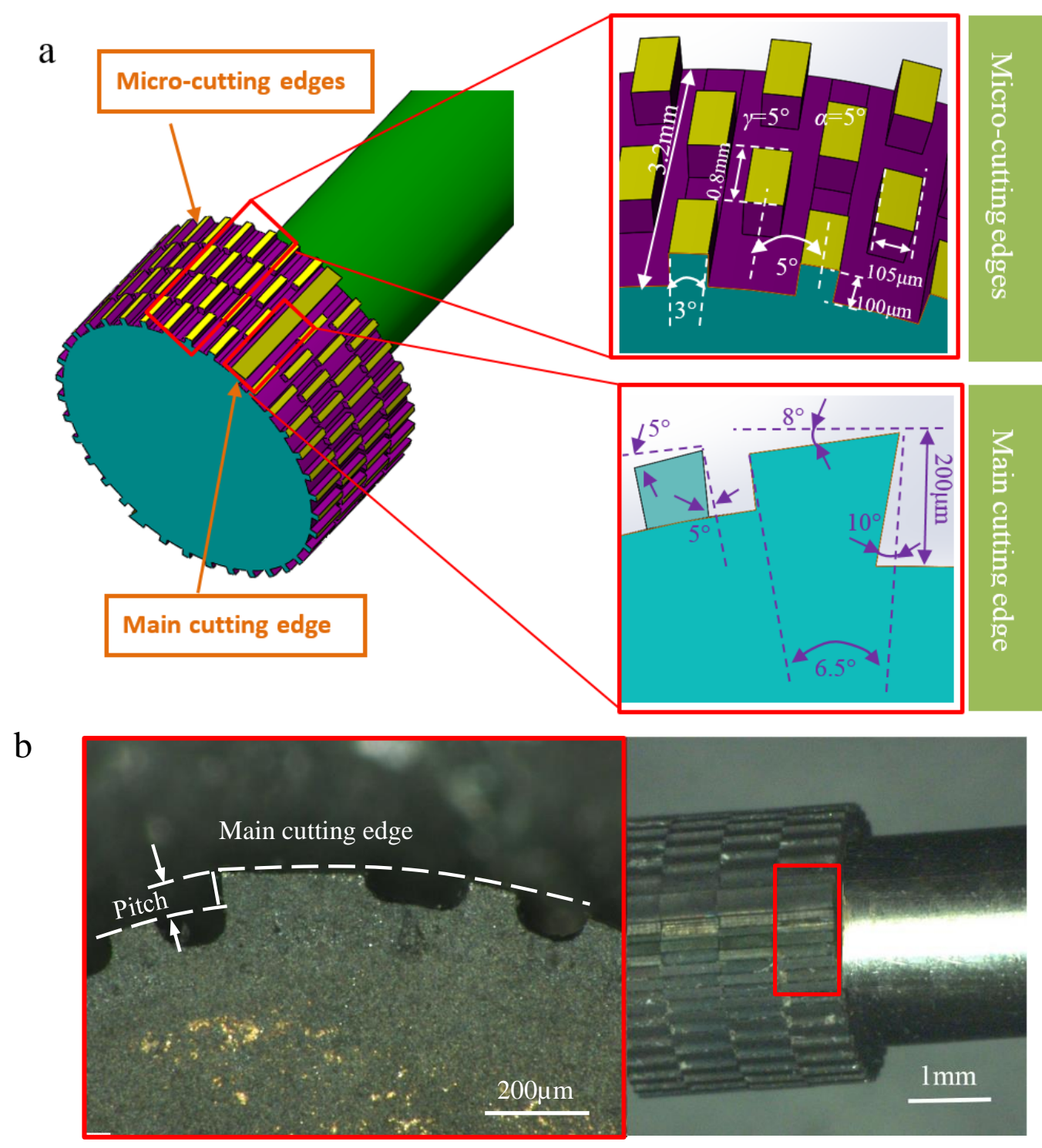




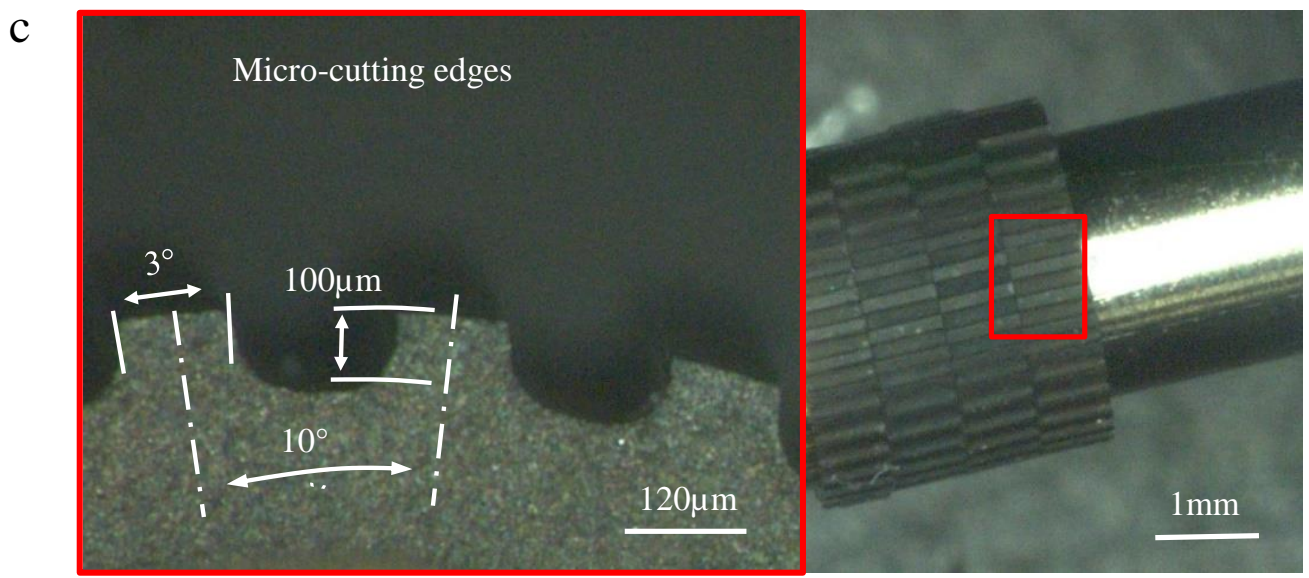

Fig. 3. Geometry design and fabrication of the proposed tool: (a) geometry design, (b) manufactured main cutting edge and (c) micro-cutting edges.

\section{Performance testing/evaluation of the proposed tool}

The performance of proposed tool was evaluated against a conventional milling cutter (cemented carbide, 2 flutes, tool diameter $4.0 \mathrm{~mm}$, rake angle $\gamma_{c o n}=10^{\circ}$, clearance angle $\alpha_{c o n}=0^{\circ}$, helix angle $\lambda_{c o n}=30^{\circ}$ ) by performing side milling tests on a 5-axis machine tool under dry cutting conditions. The workpiece used in this study was acquired from mid-diaphysis of bovine femurs since the distribution of constituents is very similar to the human bone [2]. The workpiece samples (50mm length, $2 \mathrm{~mm}$ width, $15 \mathrm{~mm}$ height), cut parallel to the bone long axis direction, were utilised on the milling experiments within 1 hour after being prepared to keep the bone in fresh condition; the sample preparation followed the previously established procedure [6]. Based on a preliminary testing programme, the same set of machining parameters has been used for both milling cutters, namely: cutting speed $v_{s}=6.28 \mathrm{~m} / \mathrm{min}$, feed rate $v_{f}=0.03 \sim 0.18 \mathrm{~m} / \mathrm{min}$, radial depth of cut $a_{e}=2 \mathrm{~mm}$ and axial depth of cut $a_{p}=2 \mathrm{~mm}$. To measure the cutting temperature, the K-type thermocouple with junction diameter of $0.05 \mathrm{~mm}$ and responses time $0.125 \mathrm{~ms}$ was inserted into the bone workpiece at the depth of $1 \mathrm{~mm}$ from the uncut surface so that the tool could cut through the thermocouple while data was collected by a 16-channel data logger (GW instruments Modeccl 100) at a sampling rate of $10 \mathrm{kHz}$. After the machining experiments, the cut surfaces were analysed by Scanning Electron Microscopy (JEOL JSM-6490LV SEM) under low vacuum condition to observe the surface damage on the bone caused by cutting process; 3D profile and surface roughness of the cut bone workpiece were also measured by surface scanner (Bruker ContourGT-K). 


\section{Results and discussion}

The mean values of surface roughness ( $\mathrm{Sa}$ ) based on five repeated measurements (Fig. 4a) and the peak values of cutting temperature (Fig. 4b) have been evaluated when using both the conventional milling cutter and the proposed tool utilised at near zero wear levels (considering the high cutting lengths expected of this tool-workpiece combinations). The surface roughness (Fig.4a) in fracture cutting mode (at $f_{z C}>f_{z T}$ ) when utilising the conventional milling tool shows a distinct increase in value (ca. 33\%) compared with shear/serrated cutting mode, which coincides to the previous research results[6] that fracture mechanism deteriorates the surface. In contrast with this, the workpiece surface roughness obtained with the proposed tool increases only slightly (ca. 10\%) at the same feed rate interval when making the transition from working only with the main edge to the situation when main \& micro-edges work in a successive manner. This is owning to the differences between the cutting mechanisms on which the conventional tool would induce cracks into surface when working under fracture cutting mode while the proposed tool suppresses the MUCT of main cutting edge to a low level (around $100 \mu \mathrm{m}$ ) while the micro-cutting edges perform a finishing action the surface due to the shear cutting working mechanism. Moreover, the cutting temperature of proposed hybrid tool has also been found to be reduced distinctly compared with the conventional tool (Fig. 4b), which is mainly due to the proposed tool geometry and the application of solid diamond material with its high thermal conductivity.

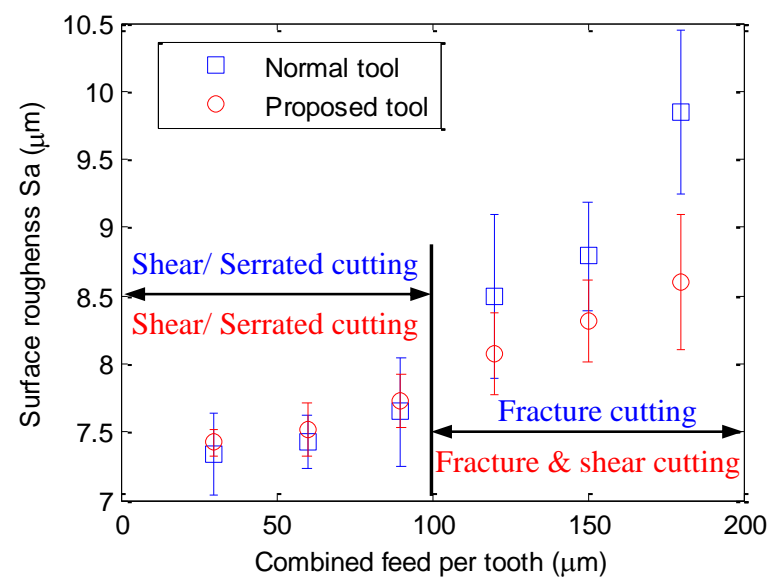

(a)



(b)

Fig. 4. Evolution of surface roughness (a) and cutting temperature (b) versus combined feed per tooth from conventional milling tool and proposed novel tool.

Scanned 3D surfaces and SEM micrographs show comparable good surface quality for conventional milling tool and the proposed tool under small values of combined feed per tooth $\left(f_{z}=30 \mu \mathrm{m}\right)$, as shown 
in Fig. 5(a) and Fig. 5(c). In this condition, the proposed tool works within main edge only cutting mode and the micro-cutting edges are not active (Fig. 2b non-working area), hence the finishing function is disabled, and the surface quality is similar to that of the commercial tool. Indeed, in this stage there is no fracture generated and the resulted shear/serrated morphology of chip leads to smooth surface of both conventional and the proposed tool.

a
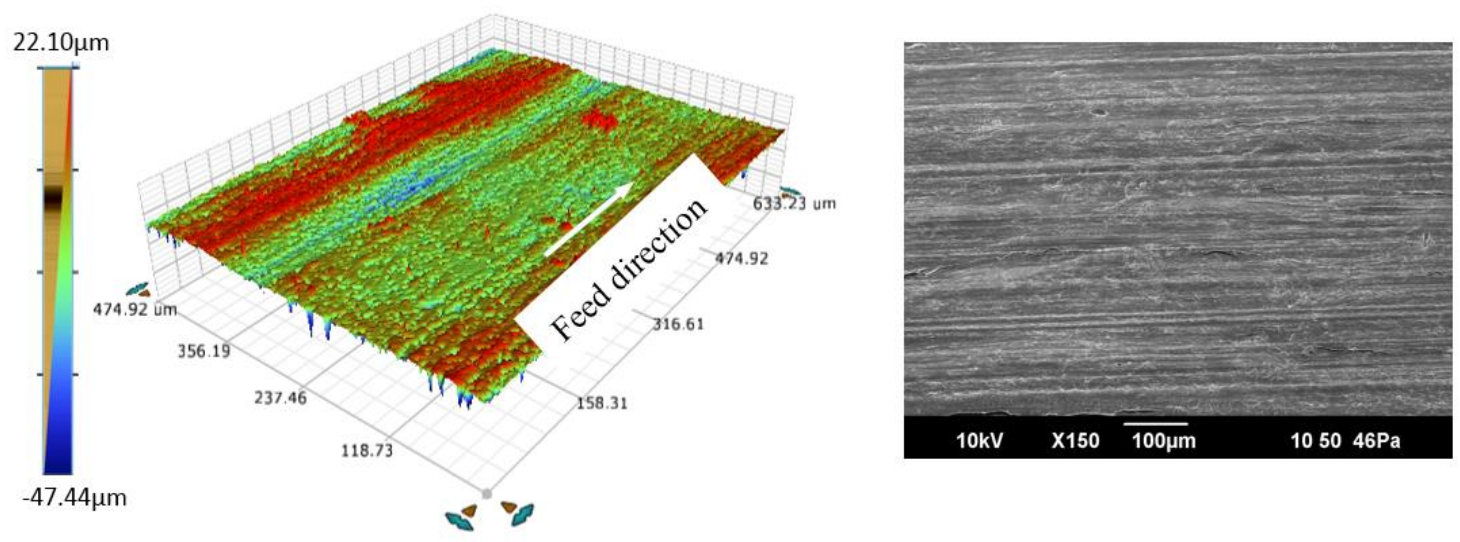

$\mathrm{b}$




Fig. 5. 3D scanned and SEM surface of bone workpiece generated from conventional milling tool under small $\left(f_{z}=30 \mu \mathrm{m}\right)$ (a) and large combined feed per tooth $\left(f_{z C}=180 \mu \mathrm{m}\right)(\mathrm{b})$ and proposed tool under small $\left(f_{z C}=30 \mu \mathrm{m}\right)(\mathrm{c})$ and large combined feed per tooth $\left(f_{z}=180 \mu \mathrm{m}\right)(\mathrm{d})$.

However, within high value of combined feed per tooth $\left(f_{z}=180 \mu \mathrm{m}\right)$ which corresponds to fracture cutting stage the proposed tool produced significantly superior surface integrity (Fig. 5d) than those of commercial tool (Fig. 5b). It could be seen that the surface produced by conventional tool generates significant void defects due to the fracture cutting mechanism where the osteon fibres would be piled up or extruded and distinct fracture of the workpiece could be found. On the contrary, the proposed novel tool generates a smoother surface by taking the advantage of functional micro-cutting edges, which suppresses the MUCT of main cutting edge to a low level while performing a finishing role in stop the cracks generated by the main cutting edges from propagating into the workpiece material. This significantly reduces the fracture cutting effect while minimising the surface damage to benefit the bone regeneration. 


\section{Conclusion}

In this paper, a new concept of tooling design with dual working modes, shear/serrated cutting (main edge only cutting) and combined fracture \& shear cutting (main and micro edge working successively), which minimises the surface damage when bone cutting is presented. The novel designed geometry with staggered array of main and micro-cutting edges generated around the periphery of the diamond structure following the Archimedes spiral obtained a shear/serrated cutting mode under small uncut chip thickness, while under large uncut chip thickness combined fracture \& shear cutting mode were achieved to reduce the fracture cutting and allow the "recovery" of workpiece quality.

For concept validation, a two main flutes tool with arrays of micro-cutting edges on the flank surface following a well-designed geometry was fabricated and tested with solid diamond structure. Compared with commercial tools that when accidentally feed rate is increased results in severe bone surface damage, the proposed tool design ensures smooth and limited surface anomalies even at high values of feed rates; this also comes with the advantage of improving material removal rate, thus reducing surgery intervention time, as well as lowering the cutting temperature with positive effect in reducing the risk of necrosis. The encouraging results underline the potential of proposed tool design concept in the application of other milling cutter tool (e.g. ball milling cutter) designing to be employed in bone or composite materials.

\section{References}

[1] S. Sezek, B. Aksakal, F. Karaca, Influence of drill parameters on bone temperature and necrosis: A FEM modelling and in vitro experiments, Comput. Mater. Sci. 60 (2012) 13-18.

[2] Z.R. Liao, D.A. Axinte, On monitoring chip formation, penetration depth and cutting malfunctions in bone micro-drilling via acoustic emission, J. Mater. Process. Technol. 229 (2016) 82-93.

[3] K. Denis, G.V. Ham, J.V. Sloten, R.V. Audekercke, G.V. Perre, J.D. Schutter, J.P. Kruth, J. Bellemans, G. Fabry, Influence of bone milling parameters on the temperature rise, milling forces and surface flatness in view of robot-assisted total knee arthroplasty, International congress series 1230 (2001) 300-306.

[4] C. Plaskos, A.J. Hodgson, K. Inkpen, R.W. McGraw, Bone cutting errors in total knee arthroplasty, J. Arthroplast. 17(6) (2002) 698-705.

[5] N. Sugita, T. Osa, M. Mitsuishi, Analysis and estimation of cutting-temperature distribution during end milling in relation to orthopedic surgery, Med. Eng. Phys. 31 (1) (2009) 101-107. 
[6] Z. Liao, D.A. Axinte, On chip formation mechanism in orthogonal cutting of bone, Int. J. Mach. Tools Manuf. 102 (2016) 41-55.

[7] N. Sugita, T. Osa, R. Aoki, M. Mitsuishi, A new cutting method for bone based on its crack propagation characteristics, CIRP Ann-Manuf. Technol. 58 (2009) 113-118.

[8] N. Sugita, K. Ishii, J. Sui, M. Terashima, Multi-grooved cutting tool to reduce cutting force and temperature during bone machining, CIRP Ann-Manuf. Technol. 63 (1) (2014) 101-104.

[9] P.W. Butler-Smith, D.A. Axinte, M Daine, Ordered diamond micro-arrays for ultra-precision grinding-an evaluation in Ti-6Al-4V, Int. J. Mach. Tools Manuf. 51 (2011) 54-66.

[10] G. Augustin, T. Zigman, S. Davila, T. Udilljak, T. Staroveski, D. Brezak, S. Babic, Cortical bone drilling and thermal osteonecrosis, Clin. Biomech. 27 (2012) 313-325.

[11] Z. Liao, D. Gao, Y. Lu, Z. Lv, Multi-scale hybrid HMM for tool wear condition monitoring, Int. J. Adv. Manuf. Technol. 84 (2016) 2437-2448

[12] N. Sugita, T. Nakano, Y. Nakajima, K. Fujiwara, N. Abe, T. Ozaki, M. Suzuki, M. Mitsuishi, Dynamic controlled milling process for bone machining, J. Mater. Process. Technol. 209 (17) (2009) 5777-5784.

[13] P.W. Butler-Smith, D.A. Axinte, M. Daine, Solid diamond micro-grinding tools: from innovative design and fabrication to preliminary performance evaluation in $\mathrm{Ti}-6 \mathrm{Al}-4 \mathrm{~V}$, Int. J. Mach. Tools Manuf. 59 (2012) 55-64.

[14] P.W. Butler-Smith, D.A. Axinte, M. Daine, A.R. Kennedy, L.T. Harper, J.F. Bucourt, R. Ragueneau, A study of an improved cutting mechanism of composite materials using novel design of diamond micro-core drills, Int. J. Mach. Tools Manuf. 88 (2015) 175-183.

[15] L. Tang, C. Tsai, W.W. Gerberich, L. Kruckeberg, D.R. Kania, Biocompatibility of chemical-vapourdeposited diamond, Biomaterials, 16 (6) (1995) 483-488.

[16] A. Krüger, Hard and soft: biofunctionalized diamond, Angewandte Chemie International Edition 45 (39) (2006) 6426-6427.

[17] T. Atkins, The Science and Engineering of Cutting, The Mechanics and Processes of Separating, Scratching and Puncturing Biomaterials, Materials and Non-metals, Elsevier Ltd, UK, 2009.

[18] C. Li, X. Lai, H. Li, J. Ni, Modelling of three-dimensional cutting forces in micro-end-milling, Journal of Micromechanics and Microengineering, 17(4) (2007) 671-678.

[19] M.E. Martellotti, An analysis of the milling process, Trans. ASME, 63 (8) (1941) 677-700. 